\title{
Numerical Simulation and Experimental Study on the Gas-Solid Coupling of the Aerostatic Thrust Bearing with Elastic Equalizing Pressure Groove
}

\author{
Zhao Xiao-long, Zhang Jun-an, Dong Hao, Fang Zhou, and Li Jun-ning \\ Mechanical and Electrical Engineering College, Xian University of Technology, Xian 710021, China \\ Correspondence should be addressed to Zhao Xiao-long; zhaoxiaolong@xatu.edu.cn
}

Received 25 April 2017; Revised 21 June 2017; Accepted 20 July 2017; Published 29 August 2017

Academic Editor: Marco Belloli

Copyright (c) 2017 Zhao Xiao-long et al. This is an open access article distributed under the Creative Commons Attribution License, which permits unrestricted use, distribution, and reproduction in any medium, provided the original work is properly cited.

Aiming at the problem of low stiffness of aerostatic bearing, according to the principle of gas-solid coupling, this paper designs a kind of aerostatic thrust bearing with elastic equalizing pressure groove (EEPG) and investigates the effect of elastic equalizing pressure groove (EEPG) on the stiffness of aerostatic bearing. According to the physical model of the bearing, one deduces the deformation control equation of the elastic equalizing pressure groove and the control equation of gas lubrication, using finite difference method to derive the control equations and coupling calculation. The bearing capacity and stiffness of aerostatic bearing with EEPG in different gas film clearance are obtained. The calculation results show that the stiffness increased by $59 \%$. The results of numerical calculation and experimental results have good consistency, proving the gas-solid coupling method can improve the bearing stiffness.

\section{Introduction}

Aerostatic bearings are widely used in the field of high speed and high accuracy due to their good performance $[1,2]$. However, because the air has obvious compressibility, aerostatic bearing is difficult to obtain high stiffness, so that its application field is confined to the condition of light load. Therefore, it is very important to improve the stiffness of the aerostatic bearing and expand its application field.

At present, there are two methods to improve the stiffness of aerostatic bearings: one way is the change of geometric structure of aerostatic bearing, increasing the supply pressure, increasing the throttle hole quantity, decreasing throttle hole diameter, and setting the equalizing pressure groove and a throttling cavity with different shapes on the aerostatic bearing surface to obtain a high stiffness [3-5]. Nishio et al. [6] studied the effect of orifice diameter less than $0.05 \mathrm{~mm}$ on the performance of aerostatic thrust bearings. They found that the smaller the diameter of the orifice, the greater the stiffness of the bearing. Hao and Jia [7] studied the effect of special-shaped throttling cavity on the performance of aerostatic bearing. Charki et al. [8] studied the performance of aerostatic bearings with multiple orifices. The results show that the performance of the bearing is not only related to the flow rate but also closely related to the number and diameter of the orifices. Belforte et al. [9] studied the influence of the bearing groove on the bearing pressure distribution, gas consumption, and stiffness. This work was also done in some studies [10-12]. Another way is through the electric or pneumatic precision electronic components to control the throttle effect of aerostatic bearings, in order to obtain high stiffness [13]. Aguirre et al. study [14] is driven by an electromagnetic drive mechanism to control the gas film clearance changes, so as to improve the bearing stiffness. Ghodsiyeh et al. [15] adopt pneumatic control as a semiactive compensation and improve the aerodynamic stiffness of the air bearing, and the main parameters affecting the static performance of the system are discussed. Compared with the above two methods, with only changing the geometry of aerostatic bearings, the structure is simple, but it is difficult to obtain a larger stiffness. Compared with the first kind of research, the second research method, through active control 


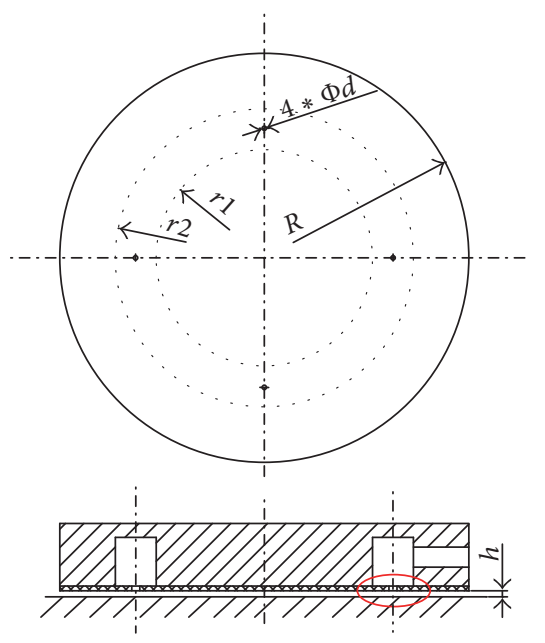

FIGURE 1: Schematic diagram of physical model.

and dynamic compensation, can get larger stiffness, but this kind of aerostatic bearing has complex structure, with high degree of mechanical and electrical integration.

Through the above analysis, it can be seen that the gas film clearance is an important factor that affects the stiffness of aerostatic bearing. In this paper, the gas-solid coupling method is proposed to enhance the stiffness of aerostatic bearing with EEPG, and the gas film clearance can be changed dynamically through the deformation of EEPG. The mathematical model of the gas-solid coupling of EEPG is derived. The numerical simulation and experimental results show that the EEPG can improve the stiffness of aerostatic bearing.

\section{Physical Model and Calculation Method}

2.1. Working Principle. This section mainly introduces the two-dimensional physical model and its working principle. This new type of bearing consists of two parts: the bearing body and the elastic thin plate. The elastic thin plate is connected with the bearing body through vacuum brazing, and the EEPG is mainly obtained by the deflection deformation of elastic thin plate in this paper. The new bearing structure is shown in Figure 1. The radius is $R$, there is an annular airway in the bearings of the internal part, and the elastic thin plate is covered on the annular airway. There are four orifices that are evenly distributed on the elastic thin plate, and the diameter is $d$. The minimum radius of the annular airway is $r_{1}$, and the maximum radius is $r_{2}$. The width value of the annular airway is the difference between the maximum radius and the minimum radius, that is, $r_{2}-r_{1}$.

Figure 2(a) is the bearing of the EEPG processing schematic. Firstly, high pressure gas is injected into the bearing to make the elastic deformation of the elastic thin plate, as shown in Figure 2(a)(1). And then grind the convex part of the elastic thin plate, as shown in Figures 2(a)(2) and 2(a)(3). Then stop gas supply, the deformation is restored, elastic plate will form a groove in an annular passage, and the concave shallow groove is the initial EEPG.
The bearing is working, the elastic thin plate is affected by the pressure difference between the supply pressure and the gas film pressure, and thin elastic deformation is generated, thus forming an EEPG. The gas film clearance is small, with gas film pressure distribution near the supply pressure, and the pressure difference of thin plate is small, so the basic elastic thin plate is not deformed. Elastic equalizing pressure groove depth of aerostatic bearing is substantially equal to the initial processing produce. At this time, the working state is equal to the working state of the aerostatic bearing of the REPG (rigid equalizing pressure groove). The gas film clearance increases to a certain range, the gas film pressure is less than the supply pressure. The elastic thin plate pressure difference increases, resulting in deformation of thin plate, but less than the depth of the initial equalizing pressure groove. At the moment, the depth of the EEPG is equal to initial elastic equalizing pressure groove minus the deformation of the thin plate. The gas film clearance increases to limit clearance, with distribution of film pressure close to standard atmospheric pressure. At the same time, the elastic thin plate is only affected by the supply pressure, the EEPG disappears, and the equalizing pressure effect disappears. Figure 2(b) shows the different working states of bearings.

It is worth noting that the gas supply pressure at work should be greater than processing pressure $\left(P_{s}>P_{s s}\right)$. In this work, the EEPG of the new aerostatic bearing will change dynamically. With the increase of the gas film clearance, the bearing capacity of the bearing decreases rapidly, and the stiffness formula $K=d F / d x$, and the faster the decrease of bearing capacity, the bigger the bearing stiffness. Compared with the aerostatic bearing with REPG, the aerostatic bearing with EEPG has better adaptability and higher stiffness.

\subsection{The Governing Equation}

2.2.1. Elastic Equalizing Pressure Groove (EEPG) Deformation Control Equation. According to the assumption of small deflection bending theory of thin plate [16], the basic equation of strain component of bending deformation can be obtained, as shown in

$$
\begin{aligned}
\varepsilon_{x} & =\frac{\partial u}{\partial x}=-z \frac{\partial^{2} w}{\partial x^{2}} \\
\varepsilon_{y} & =\frac{\partial v}{\partial y}=-z \frac{\partial^{2} w}{\partial y^{2}} \\
\gamma_{x y} & =\frac{\partial v}{\partial x}+\frac{\partial u}{\partial y}=-2 z \frac{\partial^{2} w}{\partial x \partial y}
\end{aligned}
$$

and here

$$
\begin{aligned}
& u=-z \frac{\partial w}{\partial x} \\
& v=-z \frac{\partial w}{\partial y}
\end{aligned}
$$

where $\varepsilon_{x}, \varepsilon_{y}$, and $\gamma_{x y}$ are components of strain and $w$ is Bending deflection. 


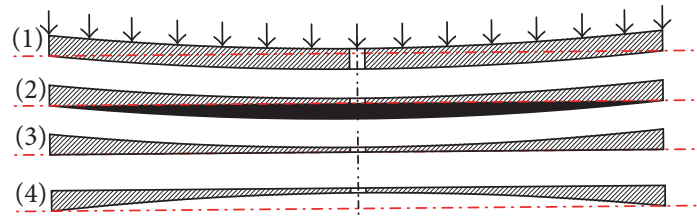

(a) Manufacture process

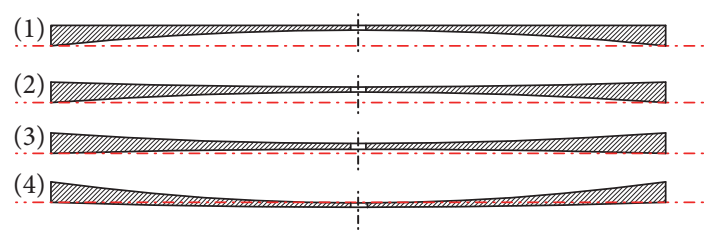

(b) Working state

Figure 2: Deformation and working state of EEPG.

The stress component is obtained by (3):

$$
\begin{aligned}
\sigma_{x} & =\frac{E}{1-\nu^{2}}\left(\varepsilon_{x}+\nu \varepsilon_{y}\right), \\
\sigma_{y} & =\frac{E}{1-\nu^{2}}\left(\varepsilon_{y}+\nu \varepsilon_{x}\right), \\
\tau_{x y} & =\frac{E}{2(1+\nu)} \gamma_{x y} .
\end{aligned}
$$

That is,

$$
\begin{aligned}
\sigma_{x} & =-\frac{E z}{1-\nu^{2}}\left[\frac{\partial^{2} w}{\partial x^{2}}+\nu \frac{\partial^{2} w}{\partial y^{2}}\right], \\
\sigma_{y} & =-\frac{E z}{1-\nu^{2}}\left[\frac{\partial^{2} w}{\partial y^{2}}+\nu \frac{\partial^{2} w}{\partial x^{2}}\right], \\
\tau_{x y} & =-\frac{E z}{1+\nu} \frac{\partial^{2} w}{\partial x \partial y},
\end{aligned}
$$

where $E$ is Young's modulus and $v$ is Poisson ratio.

Taking a parallelepiped element from the elastic thin plate, each edge length is $d x, d y, H$. On the cross section which perpendicular to the $x$ axis. The stress component $\sigma_{y}$ is synthesized as a bending moment; the stress component $\tau_{x y}$ is synthesized as torque; the stress component $\tau_{y x}$ is synthesized as a transverse shear force, as shown in

$$
\begin{aligned}
M_{x} & =\int_{-H / 2}^{H / 2} \sigma_{x} z d z=-D\left[\frac{\partial^{2} w}{\partial x^{2}}+\nu \frac{\partial^{2} w}{\partial y^{2}}\right], \\
M_{x y} & =\int_{-H / 2}^{H / 2} \tau_{x y} z d z=-D(1-v) \frac{\partial^{2} w}{\partial x \partial y}, \\
N_{x} & =\int_{-H / 2}^{H / 2} \tau_{z x} d z=-D \frac{\partial}{\partial x} \nabla^{2} w,
\end{aligned}
$$

and here

$$
D=\frac{E H^{3}}{12\left(1-v^{2}\right)},
$$

where $D$ is Bending stiffness and $H$ is elastic thin plate thickness.

By the same argument, on the cross section which is perpendicular to the $y$-axis, the stress component $\sigma_{y}$ is synthesized as a bending moment; the stress component $\tau_{x y}$ is synthesized as torque; the stress component $\tau_{y x}$ is synthesized as a transverse shear force, as shown in

$$
\begin{aligned}
M_{y} & =\int_{-H / 2}^{H / 2} \sigma_{y} z d z=-D\left[\frac{\partial^{2} w}{\partial y^{2}}+\nu \frac{\partial^{2} w}{\partial x^{2}}\right], \\
M_{x y} & =\int_{-H / 2}^{H / 2} \tau_{x y} z d z=-D(1-\nu) \frac{\partial^{2} w}{\partial x \partial y}, \\
N_{y} & =\int_{-H / 2}^{H / 2} \tau_{y x} d z=-D \frac{\partial}{\partial y} \nabla^{2} w .
\end{aligned}
$$

The equilibrium equation of thin plate is

$$
\begin{aligned}
N_{x} & =\frac{\partial M_{x}}{\partial x}+\frac{\partial M_{x y}}{\partial y}, \\
N_{y} & =\frac{\partial M_{x y}}{\partial x}+\frac{\partial M_{y}}{\partial y}, \\
\frac{\partial N_{x}}{\partial x}+\frac{\partial N_{y}}{\partial y}+q & =0 .
\end{aligned}
$$

From (5), (7), and (8) one can get the deformation control equation, as shown in

$$
\frac{\partial^{4} w}{\partial x^{4}}+2 \frac{\partial^{4} w}{\partial x^{2} \partial z^{2}}+\frac{\partial^{4} w}{\partial z^{4}}=\frac{q(x, z)}{D},
$$

where $q$ is uniform pressure.

The calculation model of this paper is a circle, so formula (9) is transformed into polar coordinates:

$$
\begin{gathered}
\frac{\partial^{4} w}{\partial r^{4}}+\frac{2}{r^{2}} \frac{\partial^{4} w}{\partial r^{2} \partial \theta^{2}}+\frac{1}{r^{4}} \frac{\partial^{4} w}{\partial \theta^{4}}+\frac{2}{r} \frac{\partial^{3} w}{\partial r^{3}}-\frac{2}{r^{3}} \frac{\partial^{3} w}{\partial r \partial \theta^{2}} \\
-\frac{1}{r^{2}} \frac{\partial^{2} w}{\partial r^{2}}+\frac{4}{r^{4}} \frac{\partial^{2} w}{\partial \theta^{2}}+\frac{1}{r^{3}} \frac{\partial w}{\partial r}=\frac{q(x, z)}{D} .
\end{gathered}
$$

2.2.2. Gas Lubrication Control Equation. The general form of the Reynolds equation is shown in

$$
\begin{aligned}
& \frac{\partial}{\partial x}\left(\frac{\rho h^{3}}{\mu} \frac{\partial p}{\partial x}\right)+\frac{\partial}{\partial y}\left(\frac{\rho h^{3}}{\mu} \frac{\partial p}{\partial y}\right) \\
& \quad=6\left[\frac{\partial}{\partial x} U(\rho h)+\frac{\partial}{\partial y} V(\rho h)+2 \frac{\partial(\rho h)}{\partial t}\right],
\end{aligned}
$$

where $h$ is gas film clearance, $\rho$ is density, and $p$ is the pressure. 
The ideal gas state equation is shown in

$$
\frac{p}{\rho}=\frac{p_{a}}{\rho_{a}}
$$

$p_{a}$ is standard atmospheric pressure, and $\rho_{a}$ is standard gas density.

$$
\begin{aligned}
& \frac{\partial}{\partial x}\left(p h^{3} \frac{\partial p}{\partial x}\right)+\frac{\partial}{\partial y}\left(p h^{3} \frac{\partial p}{\partial y}\right) \\
& \quad=6 \mu\left[\frac{\partial}{\partial x} U(p h)+\frac{\partial}{\partial y} V(p h)+2 \frac{\partial(p h)}{\partial t}\right]
\end{aligned}
$$

In low speed state, there is negligible relative sliding speed. Because the static gas bearing is calculated in this paper, the pressure does not change with time, and the timeterm is equal to 0 . Therefore, the right side of (13) is equal to 0 . Because of the existence of EEPG, the gas film clearance $h$ can not be eliminated from the Reynolds equation, and (13) is simplified and transformed it into polar coordinates:

$$
\frac{\partial p^{2}}{\partial r^{2}}+\frac{1}{r^{2}} \frac{\partial p^{2}}{\partial \theta^{2}}+\frac{1}{r} \frac{\partial p^{2}}{\partial r}+\frac{3}{h} \frac{\partial h}{\partial r} \frac{\partial p^{2}}{\partial r}+\frac{3}{h r^{2}} \frac{\partial h}{\partial \theta} \frac{\partial p^{2}}{\partial \theta}=0
$$

The mass flow rate of the bearing is equal to the mass flow of the outlet bearing.

$$
Q_{\text {in }}=Q_{\text {out }}
$$

Here,

$$
\begin{aligned}
& Q_{\text {in }}=A C_{0} \Psi \frac{P_{s}}{\sqrt{\Re T_{0}}}, \\
& Q_{\text {out }}=\frac{\gamma_{a} h^{3}}{12 \mu} \int_{0}^{2 \pi} \frac{\partial P}{\partial r} r d \theta, \\
& \Psi=\left\{2 g \frac{k}{k-1}\left[\left(\frac{p_{0}}{p_{s}}\right)^{2 / k}-\left(\frac{p_{0}}{p_{s}}\right)^{(k-1) / k}\right]\right\}^{1 / 2} \\
& \frac{p_{0}}{p_{s}} \geq\left(\frac{2}{k+1}\right)^{k /(k-1)},
\end{aligned}
$$

$$
\begin{aligned}
\Psi=\left[2 g \frac{k}{k-1}\left(\frac{2}{k+1}\right)^{2 /(k-1)}\right. & ]^{1 / 2} \\
& \frac{p_{0}}{p_{s}}<\left(\frac{2}{k+1}\right)^{k /(k-1)},
\end{aligned}
$$

where $Q_{\text {in }}$ is mass flow in, $Q_{\text {out }}$ is mass flow out, $g$ is acceleration of gravity, $\mu$ is dynamic viscosity, $P_{0}$ is outlet pressure, $P_{\mathrm{s}}$ is supply pressure, $T_{0}$ is temperature, $\mathfrak{R}$ is gas constant, $k$ is adiabatic index, $C_{0}$ is flow coefficient $d$ is orifice diameter, and $\psi$ is flow velocity coefficient.

Given the boundary conditions and the simultaneous equations (14) and (15) to obtain the numerical solution, we can get the pressure distribution of the gas film surface and calculate the bearing capacity $W$.

$$
W=\int_{0}^{R} \int_{0}^{2 \pi}\left[P(r, \theta)-P_{a}\right] r d r d \theta .
$$

The calculation formula of bearing stiffness is as follows:

$$
K=\frac{d W}{d h},
$$

where $K$ is stiffness, $d h$ is the change volume of gas film clearance, and $d F$ is the change volume of bearing capacity.

The calculation formula of stiffness is obtained:

$$
K=\frac{\left(W_{1}-W_{2}\right)}{\Delta h} \text {. }
$$

2.3. The Boundary Conditions. The EEPG generates deflection deformation in the range of $r_{1}<r<r_{2}$, when $0<r \leq r_{1}$ or $R>r \geq r_{2}$, and no elastic deformation, $w=0$.

The pressure $p_{i, j}=P_{s}$ at the orifice $(i, j)$ of the gas lubrication governing equation, the pressure $p_{i, j \mid i=m+1, j=1: n+1}=P_{a}$ at the edge of the bearing $(i, j)$.

2.4. Difference Scheme. The governing equations (10) and the governing equations (14) are discretized separately. This paper uses 2-order accuracy central difference and the control equation (10) of the 3-order and 4-order difference schemes such as formula (20). The governing equation (14) uses 1order and 2-order difference scheme, which is omitted in this paper. The 4-order finite difference scheme in polar coordinates is shown in Figure 3.

$$
\begin{aligned}
& \left.\frac{\partial^{4} w}{\partial r^{4}}\right|_{i, j}= \\
\left.\frac{\partial^{4} w}{\partial \theta^{4}}\right|_{i, j} & =\frac{w_{i+2, j}-4 w_{i+1, j}+6 w_{i, j}-4 w_{i-1, j}+w_{i-2, j}}{\Delta r^{4}}, 6 w_{i, j}-4 w_{i-1, j}+w_{i-2, j} \\
\left.\frac{\partial^{4} w}{\partial \theta^{2} \partial r^{2}}\right|_{i, j} & =\frac{w_{i+2, j+1}-2\left(w_{i, j+1}+w_{i+1, j}+w_{i-1, j}+w_{i, j-1}\right)+w_{i-1, j+1}+w_{i+1, j-1}+w_{i-1, j-1}-4 w_{i, j}}{\Delta \theta^{2} \Delta r^{2}}, \\
\left.\frac{\partial^{3} w}{\partial r^{3}}\right|_{i, j} & =\frac{w_{i+2, j}-2 w_{i+1, j}+2 w_{i-1, j}-w_{i-2, j}}{2 \Delta r 3}, \\
\left.\frac{\partial^{4} w}{\partial \theta^{2} \partial r}\right|_{i, j} & =\frac{w_{i+1, j+1}+w_{i+1, j-1}-w_{i-1, j+1}-w_{i-1, j-1}-2\left(w_{i+1, j}-w_{i-1, j}\right)}{2 \Delta \theta^{2} \Delta r},
\end{aligned}
$$




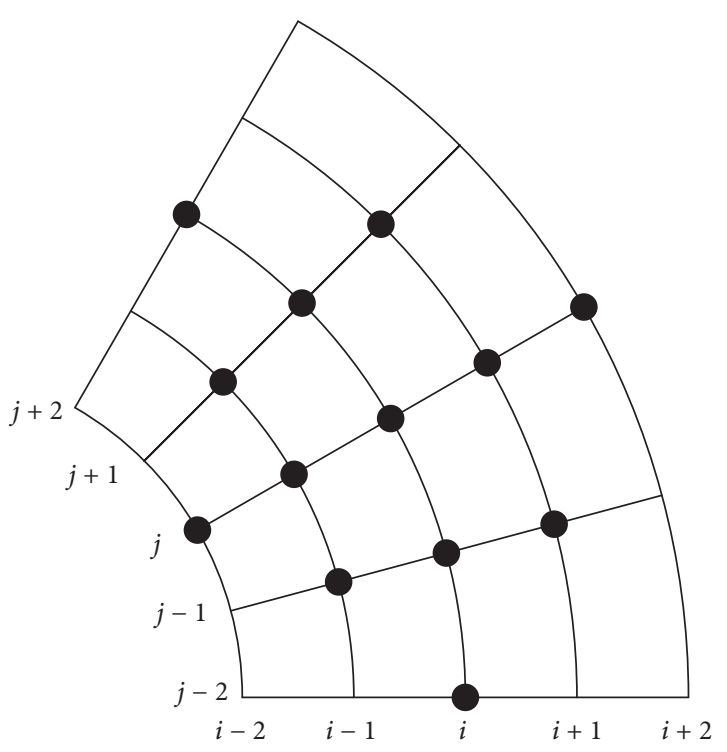

FIGURE 3: 4-order finite difference scheme in polar coordinates.

where $i, j$ are grid number in the direction of $r, \theta$, respectively; $\Delta r, \Delta \theta$ are grid distance in the direction of $r, \theta$, respectively.

2.5. Gas-Solid Coupling Calculation. The calculation process of this paper is the coupling process of the deformation control equation and the gas lubrication governing equation of EEPG. The flow chart of gas-solid coupling is shown in Figure 4. Firstly, the calculation parameters are initialized, and the boundary conditions and coupling precision $\varepsilon$ are set, according to the EEPG deformation control equation to calculate the initial deformation of EEPG. Then the pressure distribution of the gas film surface is solved by the gas lubrication control equation. In the coupling process, the coupling condition is the difference between the pressure distribution of the gas film surface and processing pressure, to solve the deformation control equation of EEPG, get the deformation of elastic thin plate after the completion of the first coupling. Then, solve the gas lubrication control equation, and the numerical values of the pressure distribution of the film surface is obtained. The EEPG is also obtained, marked as coupling-hl. Set the results of the first coupling output as the initial conditions, and then solve elastic pressure groove deformation control equation and gas lubrication control equation again, the numerical values of the pressure distribution of the film surface and EEPG are obtained. The value of EEPG is coupling- $h 2$; the difference between the value of the EEPG of the two coupling outputs is used to judge the convergence condition $\Delta h=\mid$ coupling- $h 1-$ coupling- $h 2 \mid$. If $\Delta h \leq \varepsilon$, coupling ends; if $\Delta h>\varepsilon$, the calculation returns to the beginning of the first coupling. Through several iterations, until the error is less than the coupling accuracy, the coupling process is completed, and the numerical results of the EEPG after the gas-solid coupling are given. Finally, the gas lubrication control equation is solved, and the pressure
TABLE 1: Bearing parameters.

\begin{tabular}{lc}
\hline Parameter & Value \\
\hline$\mu\left(\mathrm{Kg} \cdot \mathrm{m}^{-3}\right)$ & $1.883 \times 10^{-4}$ \\
$E(\mathrm{GPa})$ & 1.12 \\
$P_{s s}(\mathrm{MPa})$ & 0.4 \\
$H(\mathrm{~mm})$ & 0.25 \\
$R(\mathrm{~mm})$ & 30 \\
$r_{1}(\mathrm{~mm})$ & 15 \\
$r_{2}(\mathrm{~mm})$ & 25 \\
$d(\mathrm{~mm})$ & 0.3 \\
$C_{0}$ & $C_{0}=0.85\left(1-e^{-8.2(w+h) / d}\right)[17]$ \\
$k$ & 1.4 \\
$R$ & 29.27 \\
$T_{0}(K)$ & 300 \\
$P_{s}(\mathrm{MPa})$ & 0.5 \\
$P_{a}(\mathrm{MPa})$ & 0.1 \\
\hline
\end{tabular}

distribution of gas film surface is obtained; calculate bearing capacity and stiffness.

\section{Test Rig}

Build the aerostatic bearing stiffness test rig, as shown in Figure 5. The experimental bench is loaded by the jack, and the load is transmitted to the tested bearing through the force sensor and the force transmission rod, and the working gas film clearance of the bearing is measured by the microdisplacement sensor. The maximum output pressure of the test bench is $5000 \mathrm{~N}$, and the measurement accuracy of the microdisplacement sensor is $0.1 \mu \mathrm{m}$.

\section{Results and Discussion}

4.1. Theoretical Result. According to the calculation method of the previous section, the calculation parameters of gas bearing, as shown in Table 1, are selected to calculate the gas-solid coupling. The deformation curves of the EEPG and the film pressure distribution of the aerostatic bearing are obtained under different gas film clearance, as shown in Figure 6, where (i) is the elastic pressure groove coupling deformation graph and (ii) is the gas film pressure distribution. Suppose that this calculation is a computational model 1.

In order to compare the performance of the aerostatic bearing with EEPG, this paper calculated the performance of aerostatic bearing with REPG in the same performance parameters. Calculation parameters are also shown in Table 1, and the depth value of the pressure equalizing groove does not change with the pressure. At this time, we can obtain the film pressure distribution of the aerostatic bearing with REPG by solving (12), as shown in Figure 7. Suppose that this calculation is a computational model 2 .

According to the calculation results of model 1 and model 2, the numerical performance curves of the 2 kinds 


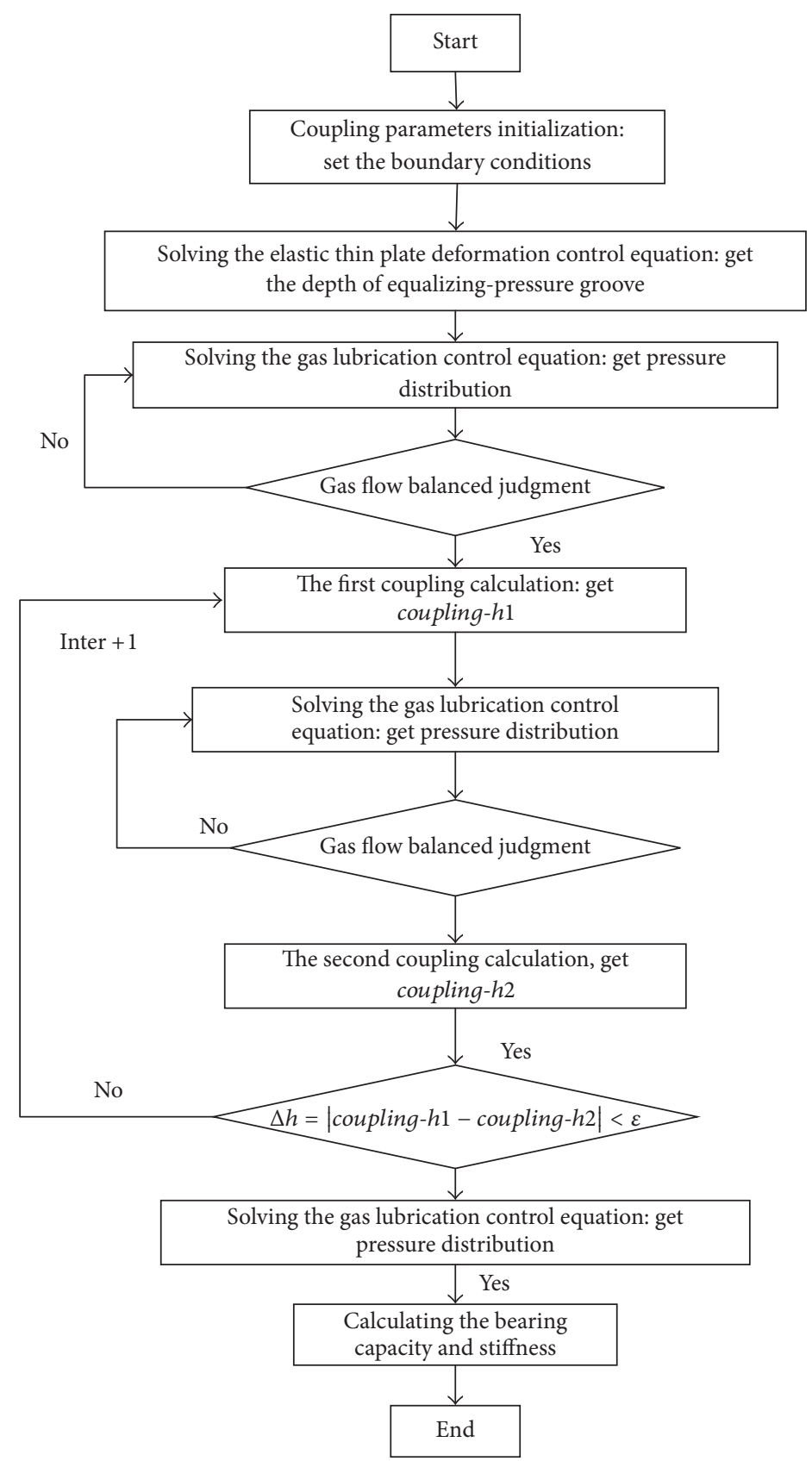

FIGURE 4: Flow chart of gas-solid coupling.

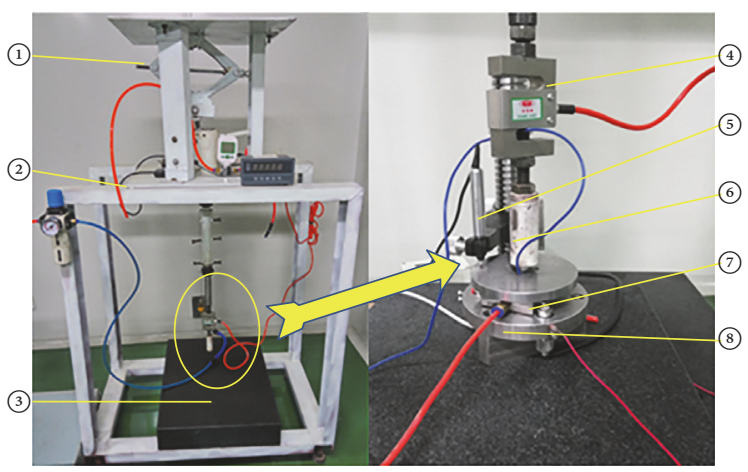

Figure 5: Bearing stiffness test rig. (1) Lifting jack. (2) Test bench. (3) Granite pedestal. (4) Force sensor. (5) Microdisplacement sensor. (6) Support bars. (7) Tested aerostatic bearing. (8) Bearing platform. 


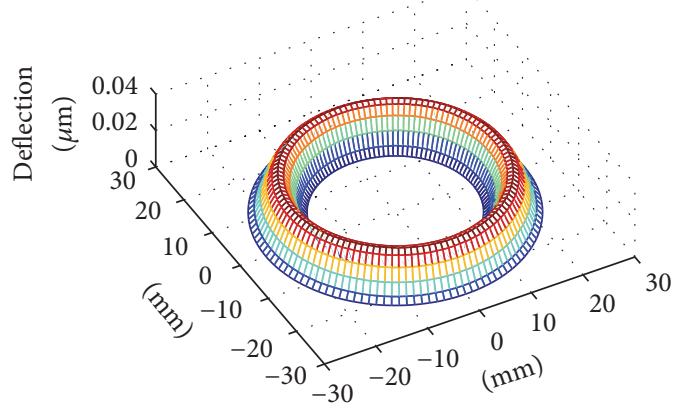

(i)

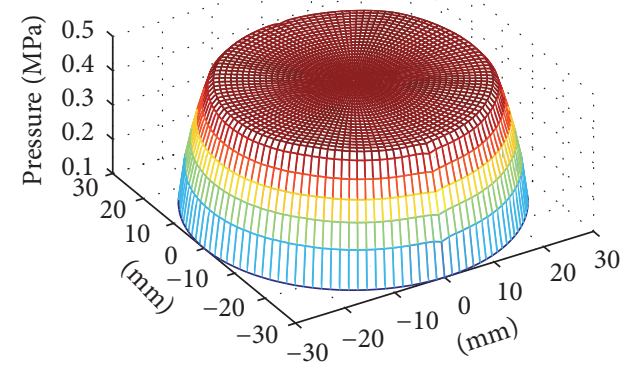

(ii)

(a) $h=5 \mu \mathrm{m}$

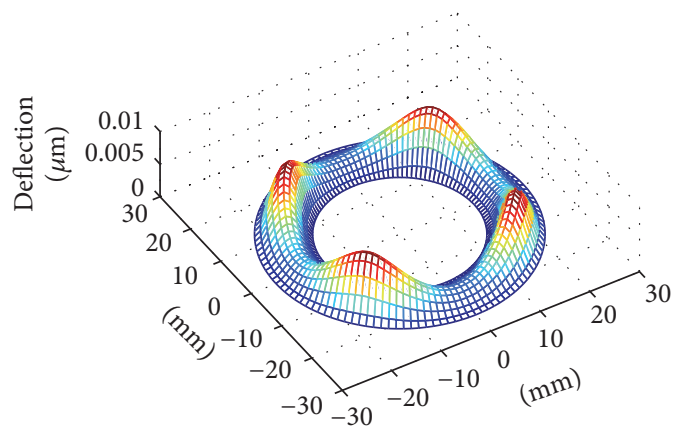

(i)

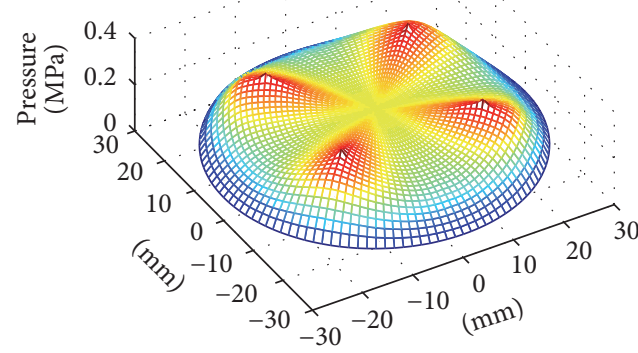

(ii)

(b) $h=8 \mu \mathrm{m}$

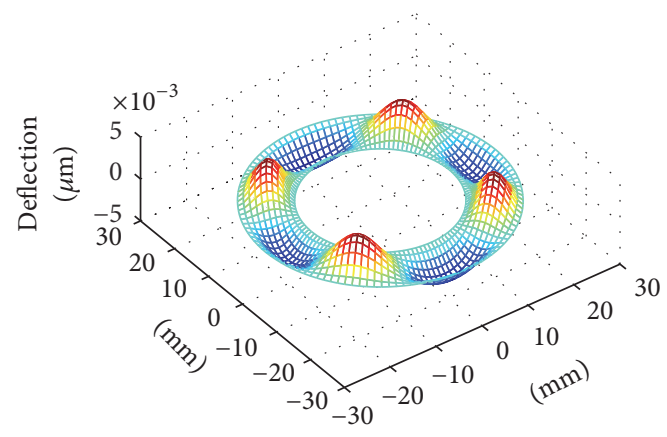

(i)

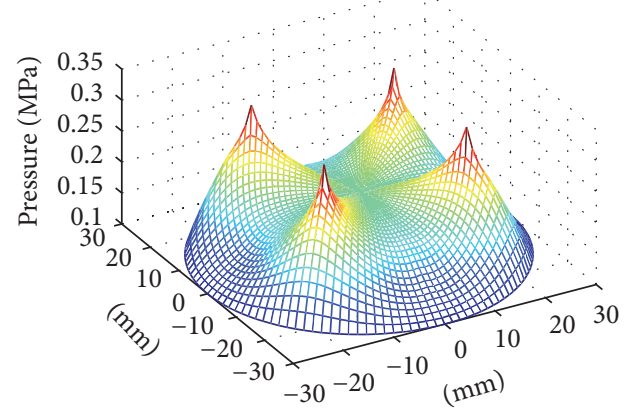

(ii)

(c) $h=11 \mu \mathrm{m}$

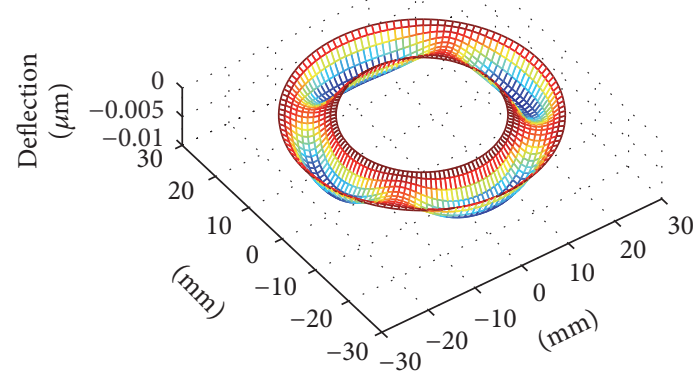

(i)

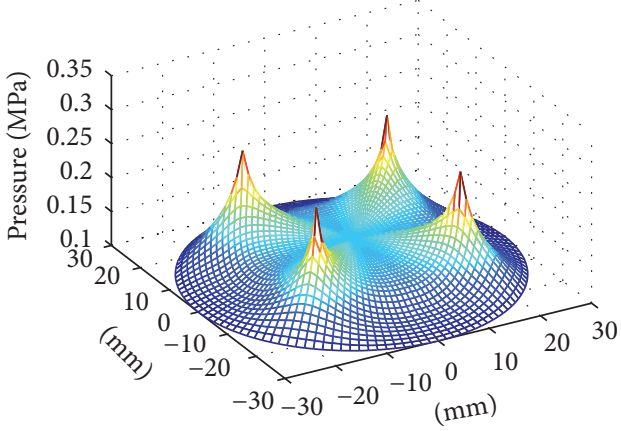

(ii)

(d) $h=16 \mu \mathrm{m}$

FIGURE 6: Diagram of (i) coupling deformation of EEPG and (ii) pressure distribution. 


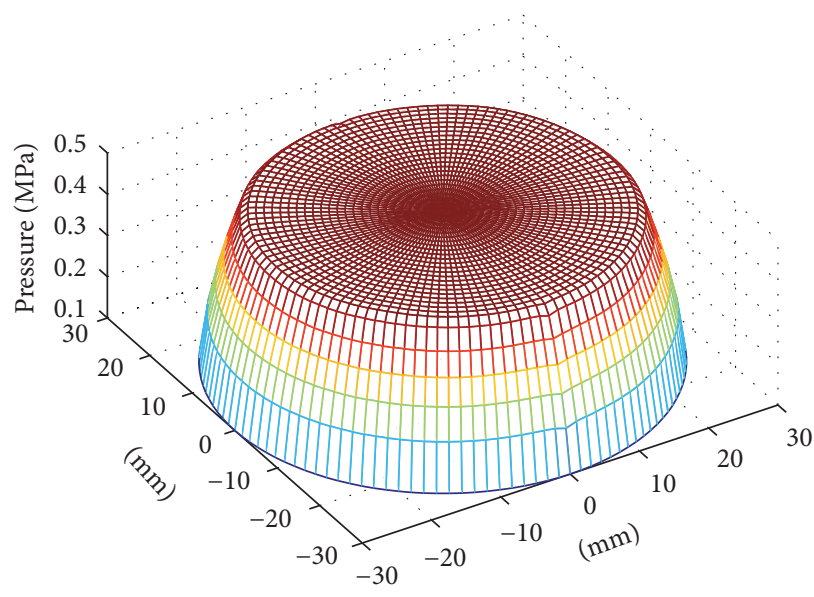

(a) $h=5 \mu \mathrm{m}$

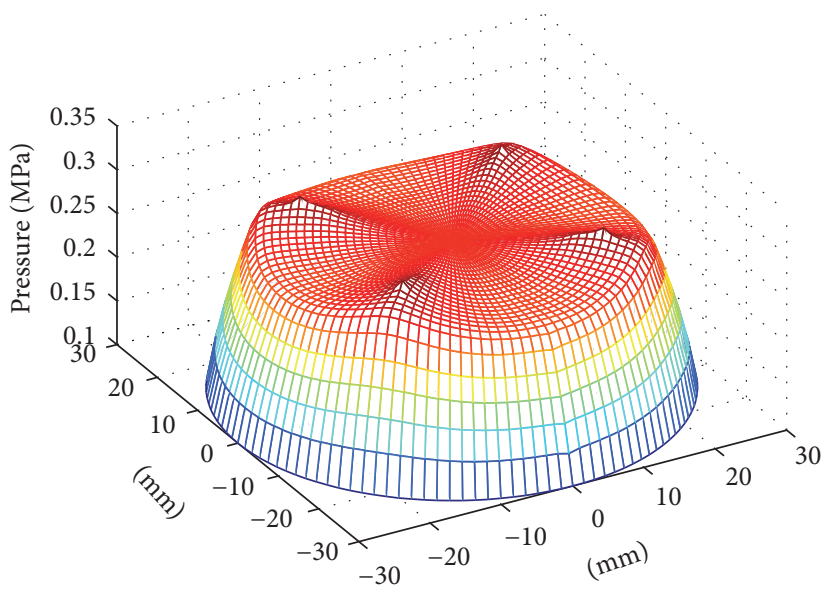

(c) $h=11 \mu \mathrm{m}$

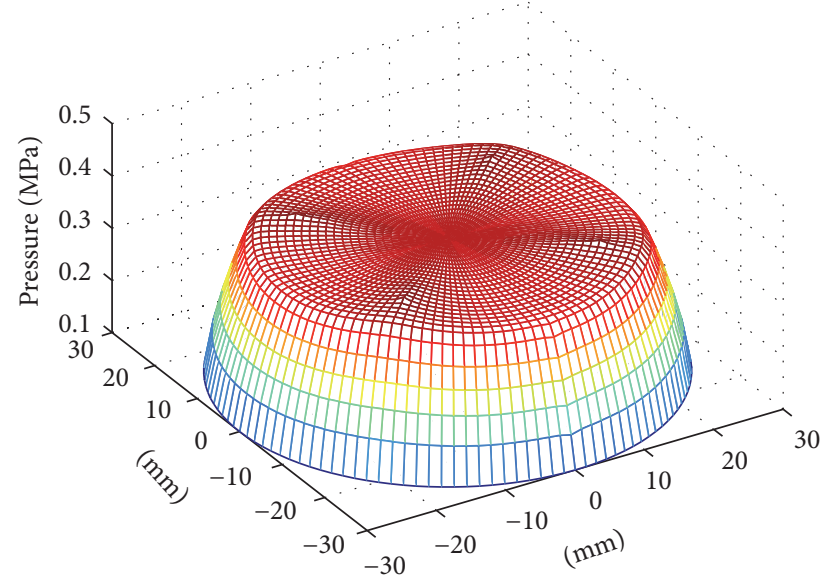

(b) $h=8 \mu \mathrm{m}$

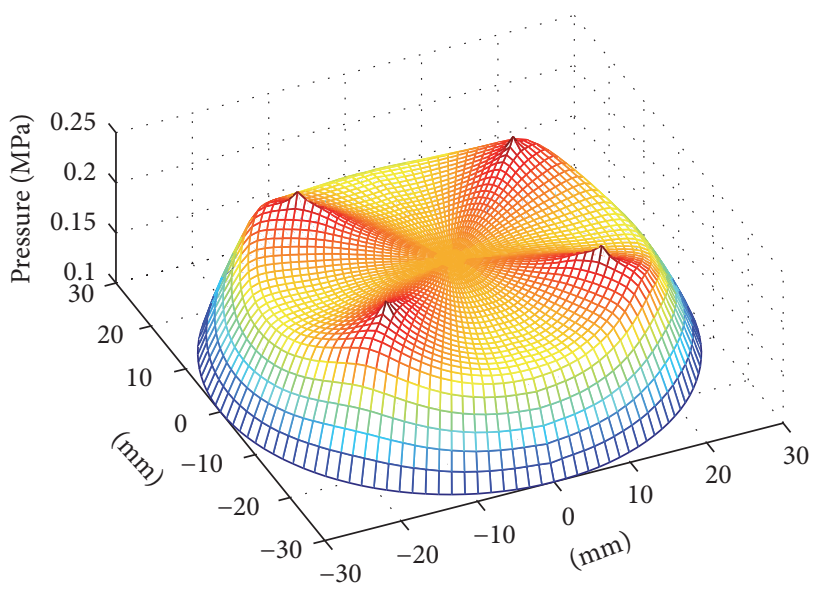

(d) $h=16 \mu \mathrm{m}$

FIGURE 7: Pressure distribution of aerostatic bearing with REPG.

of aerostatic bearings can be obtained, as shown in Figure 8, where model 1 is the aerostatic bearing with EEPG and model 2 is the aerostatic bearing with REPG. Figure $8(a)$ is the bearing capacity comparison curve, Figure $8(\mathrm{~b})$ is the stiffness contrast curve, and Figure 8(c) is a gas flow rate curve.

It can be seen from Figure 8(a) that the bearing capacity decreases with the increase of gas film clearance. The bearing capacity of model 1 overall compared to model 2 bearing capacity is small, but when the gas film clearance is less than or equal to $5 \mu \mathrm{m}$ ( $h \leq 5 \mu \mathrm{m})$, model 1 and model 2 have the same bearing capacity. This is because when the gas film clearance is small, the pressure on the two sides of the elastic thin plate of model 1 are nearly equal, and the pressure difference is approximately equal to 0 . Therefore, the elastic pressure groove has almost no deformation, as shown in Figure 6(a)(i), and the gas film pressure distribution is shown in Figure 6(a)(ii).

When the gas film clearance is from $6 \mu \mathrm{m}$ to $15 \mu \mathrm{m}(15 \mu \mathrm{m}$ $>h>6 \mu \mathrm{m})$, the bearing capacity of model 1 is less than that of model 2 . Because the air supply remained unchanged, with the gas film clearance increases, the bearing capacity is decreased. Thus, the pressure difference between the upper and the lower surfaces of the elastic thin plate is increased, and the EEPG is forced to deform outwards, the sealing ability of the EEPG decreases, and the bearing capacity decreases rapidly. The deformation of the EEPG is shown in Figures 6(b)(i) and 6(c)(i). The deformation of the EEPG is uneven, and the maximum deformation occurs between the 2 orifices. The gas film pressure distribution is shown in Figures 6(b)(ii) and $6(\mathrm{c})$ (ii).

When the gas film clearance is more than $16 \mu \mathrm{m}(h>$ $16 \mu \mathrm{m})$, model 1 bearing capacity is much smaller than model 2. Because the pressure difference of the elastic thin plate is greater than the initial working pressure, the deformation of EEPG is greater than the initial EEPG deformation, so the EEPG sealing effect disappeared, and bearing force decreased rapidly. The deformation of the EEPG is shown in Figure $6(\mathrm{~d})(\mathrm{i})$, and the gas film pressure distribution is shown in Figure 6(d)(ii) $(h=16 \mu \mathrm{m})$.

As shown in Figure 8(b), the stiffness of model 1 and model 2 increases first and then decreases with the increase of gas film clearance. The maximum stiffness of model 1 is in the film gap $h=7 \mu \mathrm{m}$, and the maximum stiffness of model 1 is 139 . The maximum stiffness of model 2 is in the film gap 


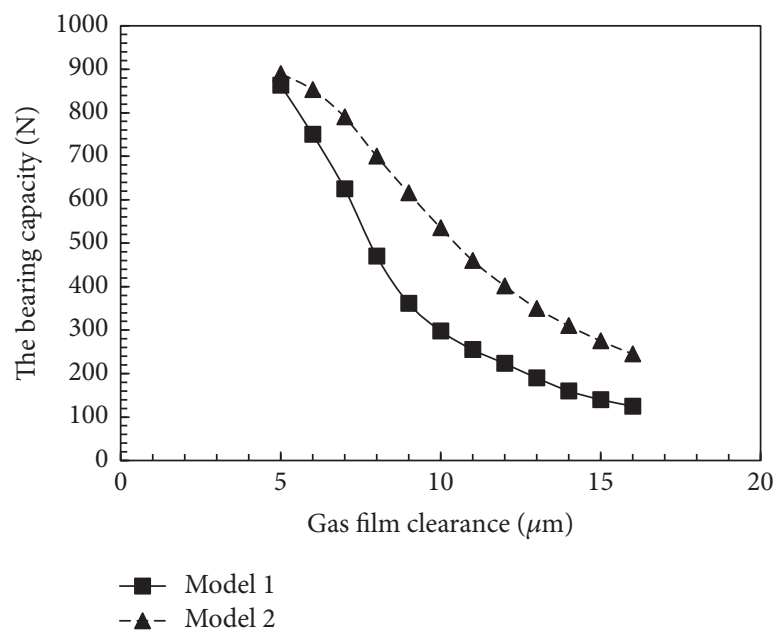

(a) Bearing capacity curve

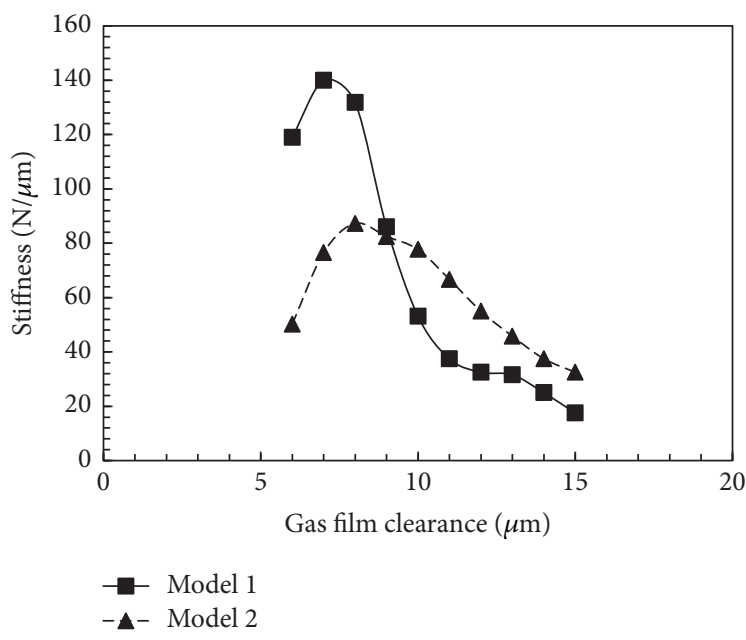

(b) Stiffness curve

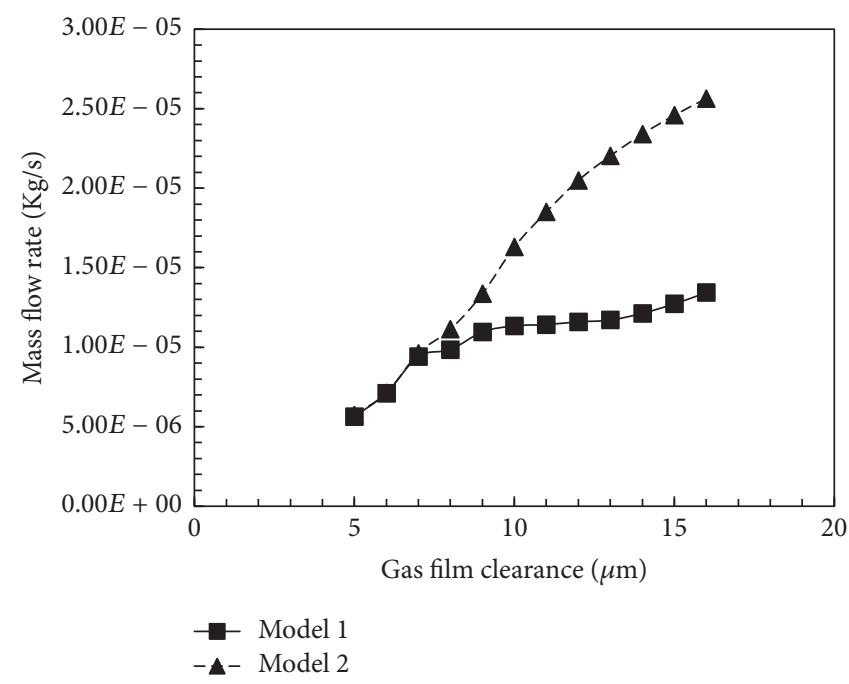

(c) Mass flow curve

FIGURE 8: Performance comparison curves of two kinds of aerostatic bearing with different pressure equalizing grooves.

$h=8 \mu \mathrm{m}$, and the maximum stiffness of model 1 is 87 . Model 1 stiffness increases compared to model 2 by $59.7 \%$, but when the gas film clearance is greater than $9 \mu \mathrm{m}$, the stiffness of model 2 is greater than that of model 1.

It can be seen from Figure 8(c) that the mass flow rate of the bearing increases with the increase of the gas film clearance. When the gas film clearance is $h>7 \mu \mathrm{m}$, the mass flow of model 1 is much less than that of model 2. This is the result of the gas-solid coupling of EEPG.

According to the above analysis, it is found that the performance of aerostatic thrust bearing with EEPG is better than that of the aerostatic bearing with REPG.

4.2. Experimental Result. Figure 9 is the experimental test bearing, processing parameters as shown in Table 1, the bearing diameter is equal to $60 \mathrm{~mm}$, and orifice diameter is equal to $0.3 \mathrm{~mm}$. The experimental bearings were treated by grinding and polishing, and the surface roughness $R_{a}$ was less than $0.2 \mu \mathrm{m}$, but the region of EEPG was not included.
The experimental results are shown in Figure 10. The results of numerical calculation and experimental test results are basically the same. The gas film clearance increases, and the bearing capacity decreased rapidly. The experimental results show that the maximum stiffness of the bearing with EEPG is obtained at $h=7 \mu \mathrm{m}$ and is consistent with the numerical calculation and well validated the numerical gassolid coupling calculation is correct. However, the bearing capacity of the test bearing is less than the bearing capacity of the numerical calculation, because of the influence of the surface roughness of the test bearing, the position accuracy of the orifice, and the grinding precision of the EEPG.

\section{Conclusion}

In this paper, the bearing characteristics of aerostatic bearing with EEPG are studied by numerical calculation and experiment. Through the research, we can draw the following conclusions. 


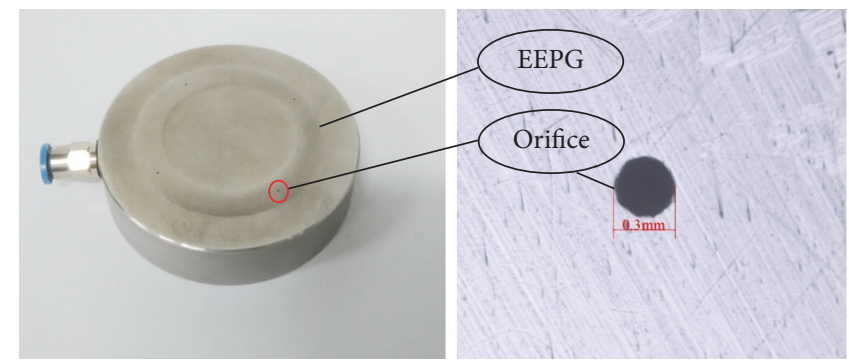

FIgURE 9: Tested aerostatic bearings.

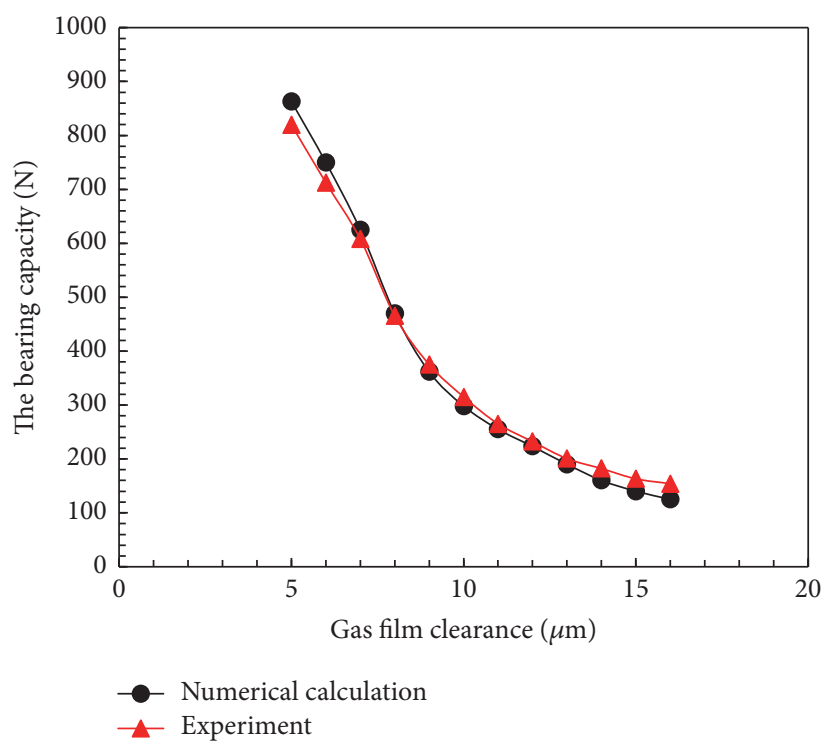

(a) Bearing capacity curve

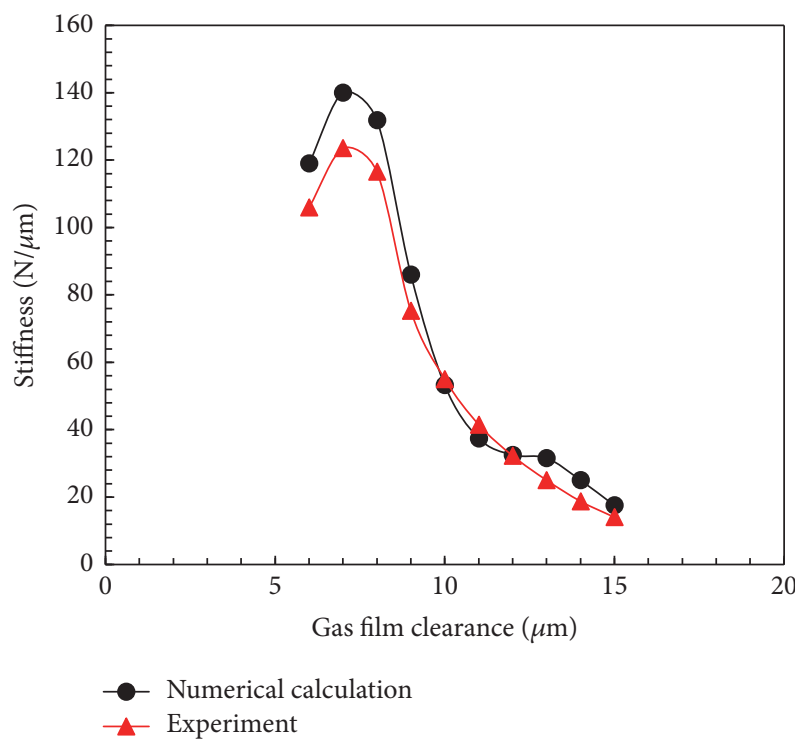

(b) Stiffness curve

Figure 10: Experimental results.

(1) In this paper, the use of gas-solid coupling adaptive mode of aerostatic bearing is feasible in theory and practice.

(2) The stiffness of aerostatic thrust bearing with EEPG is increased by $40 \%$ than that of the aerostatic bearing with REPG.

(3) The structure and location of the EEPG have a great influence on the performance of the bearing, so how to optimize the design warrants further research.

\section{Conflicts of Interest}

The authors declare that they have no conflicts of interest.

\section{Acknowledgments}

The authors would like to acknowledge that the project was supported by the National Natural Science Foundation of China (51375384), China Postdoctoral Fund (2016M602937XB), and Xi'an Technological University gas lubrication research and innovation team construction project.

\section{References}

[1] J. Du, G. Zhang, T. Liu, and S. To, "Improvement on load performance of externally pressurized gas journal bearings by opening pressure-equalizing grooves," Tribology International, vol. 73, no. 5, pp. 156-166, 2014.

[2] S. Gao, K. Cheng, S. Chen, H. Ding, and H. Fu, "CFD based investigation on influence of orifice chamber shapes for the design of aerostatic thrust bearings at ultra-high speed spindles," Tribology International, vol. 92, pp. 211-221, 2015.

[3] Y. F. Li, Y. H. Yin, H. Yang, X. N. Liu, J. Mo, and H. L. Cui, "Modeling for optimization of circular flat pad aerostatic bearing with a single central orifice-type restrictor based on CFD simulation," Tribology International, vol. 109, pp. 206-216, 2017.

[4] Y. R. Jeng and S. H. Chang, "Comparison between the effects of single-pad and double-pad aerostatic bearings with pocketed orifices on bearing stiffness," Tribology International, vol. 66, no. 7, pp. 12-18, 2013.

[5] J.-A. Zhang, H. Dong, F.-J. Ma, and B. Liu, "Performance analysis of the flotation cushion with elastic pressure equalizing groove for aerostatic slideway," Applied Mechanics and Materials, vol. 152-154, pp. 743-748, 2012. 
[6] U. Nishio, K. Somaya, and S. Yoshimoto, "Numerical calculation and experimental verification of static and dynamic characteristics of aerostatic thrust bearings with small feedholes," Tribology International, vol. 44, no. 12, pp. 1790-1795, 2011.

[7] A. P. Hao and Y. H. Jia, "Research on the characteristics of bearing behavior of the profiled throttling cavity air bearing," in Proceedings of the 7th Asian-Pacific Conference on Aerospace Technology and Science, APCATS 2013, pp. 367-377, May 2013.

[8] A. Charki, K. Diop, S. Champmartin, and A. Ambari, "Numerical simulation and experimental study of thrust air bearings with multiple orifices," International Journal of Mechanical Sciences, vol. 72, no. 1, pp. 28-38, 2013.

[9] G. Belforte, F. Colombo, T. Raparelli, A. Trivella, and V. Viktorov, "Comparison between grooved and plane aerostatic thrust bearings: Static performance," Meccanica, vol. 46, no. 3, pp. 547-555, 2011.

[10] T.-J. Zi, J.-A. Zhang, Z.-W. Lu, and B. Liu, "Numerical analysis of sewing air floating needle bar based on slit type guide rail," Applied Mechanics and Materials, vol. 670-671, pp. 866-872, 2014.

[11] H. Dong, X.-L. Zhao, and J.-A. Zhang, "Static characteristic analysis and experimental research of aerostatic thrust bearing with annular elastic uniform pressure plate," Advances in Mechanical Engineering, vol. 7, no. 3, pp. 1-13, 2015.

[12] S. H. Chang, C. W. Chan, and Y. R. Jeng, "Numerical analysis of discharge coefficients in aerostatic bearings with orifice-type restrictors," Tribology International, vol. 90, pp. 157-163, 2015.

[13] T. Raparelli, V. Viktorov, F. Colombo, and L. Lentini, "Aerostatic thrust bearings active compensation: Critical review," Precision Engineering, vol. 44, pp. 1-12, 2016.

[14] G. Aguirre, F. Al-Bender, and H. Van Brussel, "A multiphysics model for optimizing the design of active aerostatic thrust bearings," Precision Engineering, vol. 34, no. 3, pp. 507-515, 2010.

[15] D. Ghodsiyeh, F. Colombo, T. Raparelli, A. Trivella, and V. Viktorov, "Diaphragm valve-controlled air thrust bearing," Tribology International, vol. 109, pp. 328-335, 2017.

[16] H. W. Liu, Theory of plate and shells, Zhejiang University Press, 1987.

[17] G. Belforte, T. Raparelli, V. Viktorov, and A. Trivella, "Discharge coefficients of orifice-type restrictor for aerostatic bearings," Tribology International, vol. 40, no. 3, pp. 512-521, 2007. 


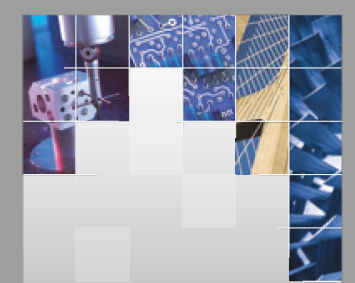

\section{Enfincering}
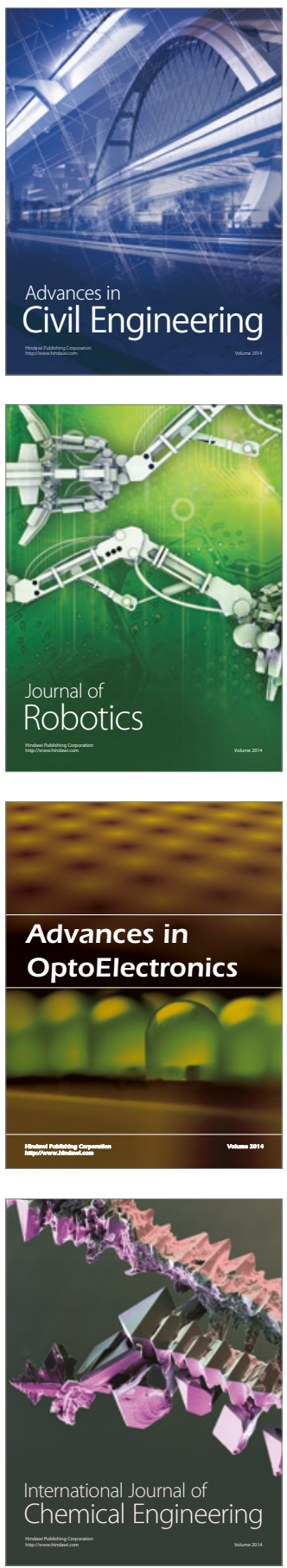

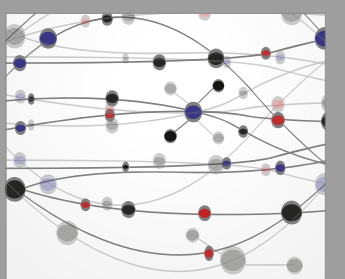

The Scientific World Journal

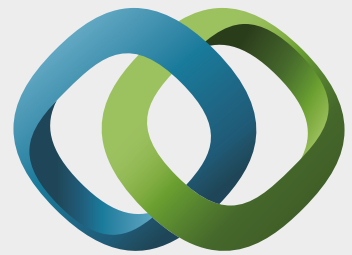

\section{Hindawi}

Submit your manuscripts at

https://www.hindawi.com
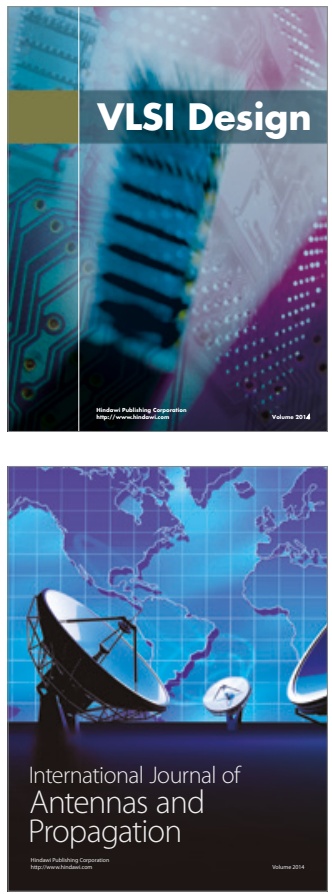

\section{Rotating}

Machinery
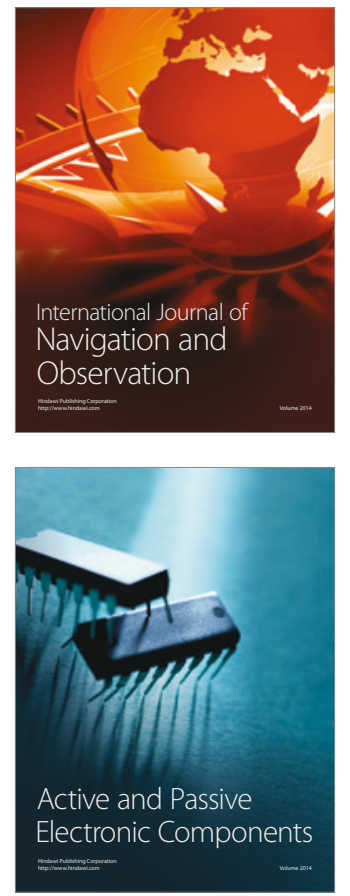
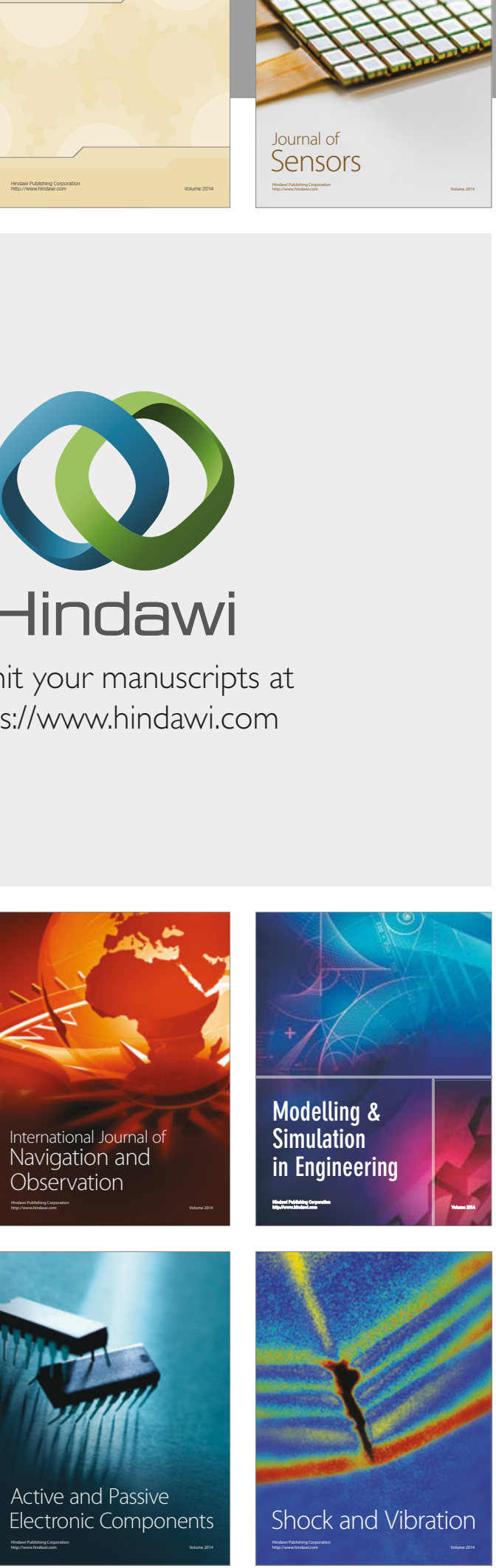
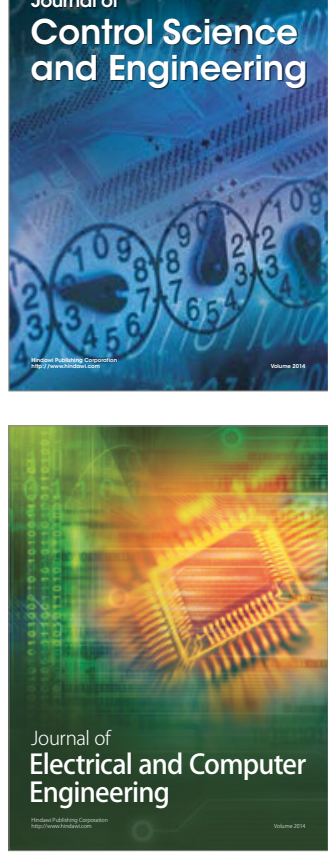

Distributed

Journal of

Control Science

and Engineering
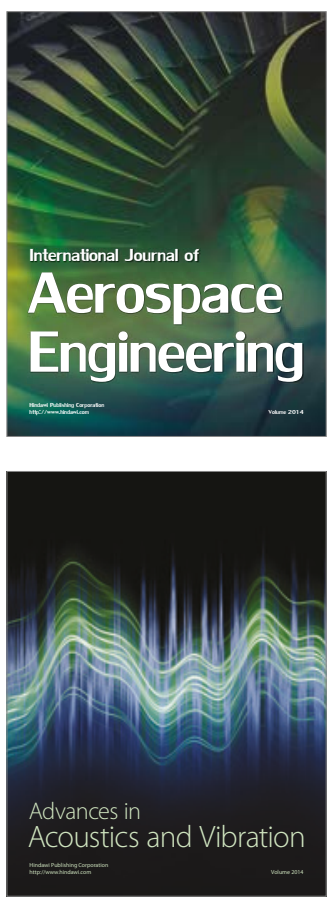

Sensor Networks 